\title{
OPA1 downregulation is involved in sorafenib-induced apoptosis in hepatocellular carcinoma
}

\author{
Xiangxuan Zhao ${ }^{1,4}$, Changhai Tian ${ }^{2,4}$, William M Puszyk ${ }^{1}$, Olorunseun O Ogunwobi ${ }^{1}$, Mengde Cao $^{3}$, Ton Wang ${ }^{1}$, \\ Roniel Cabrera ${ }^{3}$, David R Nelson ${ }^{3}$ and Chen Liu ${ }^{1}$
}

Sorafenib has been used to treat advanced hepatocellular carcinoma (HCC), but the underlying molecular mechanisms remain controversial and why some patients do not respond to this therapy is poorly understood. In this study, we show that sorafenib triggers cell growth inhibition and apoptosis in HCC cells by directly targeting the mitochondria. Treatment with sorafenib induces rapid mitochondrial fragmentation, which is associated with the deregulation of mitochondria fusion-related protein optic atrophy 1 (OPA1). Exposure of cells or isolated mitochondria to sorafenib substantially induces cytochrome $c$ release. Our data indicate that siRNA-mediated OPA1 knockdown significantly sensitizes HCC cells to sorafenib-induced apoptosis. Furthermore, sorafenib has no apparent apoptotic toxicity to normal human primary hepatocytes. Sorafenib inhibits HCC xenograft tumor growth in vivo and murine xenograft tumor tissue analysis reveals mitochondria fusion protein. OPA1 expression levels are strongly downregulated by sorafenib treatment. Western blotting evaluation of patient HCC with matched non-tumor tissue samples demonstrates that OPA1 expression is decreased in up to $40 \%$ of HCC patients. Taken together, we have shown that sorafenib suppresses the tumorigenesis of $\mathrm{HCC}$ through the induction of mitochondrial injury via OPA1. Our results provide new insights into the pathogenesis of HCC and suggest that OPA1 is a novel therapeutic target in patients with HCC.

Laboratory Investigation (2013) 93, 8-19; doi:10.1038/labinvest.2012.144; published online 29 October 2012

KEYWORDS: apoptosis; liver cancer; mitochondria fragmentation; OPA1; sorafenib

Hepatocellular carcinoma (HCC) is one of the five most common cancers worldwide. The 5-year survival rate of diagnosed HCC patients is $<10 \%{ }^{1-2}$ Therefore, new therapeutic strategies are urgently needed. Sorafenib was initially identified as a Raf (serine/threonine-specific protein kinase) and multikinase inhibitor, which targets Raf, ${ }^{3}$ Fms-like tyrosine kinase $3,{ }^{4}$ c-Kit (CD117), ${ }^{5}$ p38 (mitogen-activated protein kinase), ${ }^{6}$ stem cell growth factor receptor $1,{ }^{7}$ vascular endothelial growth factor receptors, ${ }^{8}$ and platelet-derived growth factor receptor- $\beta^{9}$ to induce growth arrest or apoptosis in a variety of malignant tumor cells. It has been reported that sorafenib treatment induces apoptosis in HepG2 cells through induction of growth arrest and DNA damage $45 \beta$ (GADD-45B). ${ }^{10}$ Other groups have demonstrated that pretreatment with the B-cell lymphoma 2 inhibitor (Bcl-2) ABT-73711 or Let-7 microRNAs, ${ }^{12}$ which block B-cell lymphoma-extra large (Bcl-xL) activity, can enhance sorafenib-induced apoptosis in HepG2 cells. Thus, the precise molecular mechanism by which sorafenib exerts its effect remains controversial. Although recent clinical studies have shown that sorafenib could inhibit HCC growth and increase the survival of patients, the efficacy of sorafenib treatment is variable and many patients do not respond well to treatment. ${ }^{13-16}$ Elucidating the molecular targets of sorafenib in HCC cells that exhibit sorafenib-induced apoptosis will provide useful clues for the identification of patients who will respond well to sorafenib treatment. Using a hepatoma cell line LH86 derived from a well-differentiated HCC in combination with an in vivo murine model of tumorigenesis, we aim to elucidate the mechanisms by which sorafenib inhibits HCC.

Abnormalities in liver mitochondrial metabolism have been found in patients with a variety of liver diseases including

\footnotetext{
${ }^{1}$ Departments of Pathology, Immunology, and Laboratory Medicine, University of Florida College of Medicine, Gainesville, FL, USA; ${ }^{2}$ Department of Pharmacology and Experimental Neuroscience, University of Nebraska Medical Center, Omaha, NE, USA and ${ }^{3}$ Department of Medicine, College of Medicine, University of Florida, Gainesville, FL, USA

Correspondence: Dr C Liu, MD, PhD, Department of Pathology, Immunology, and Laboratory Medicine, University of Florida, 1600 SW Archer Road, M651, PO Box 100275, Gainesville, FL 32610, USA.

E-mail: liu@pathology.ufl.edu

${ }^{4}$ These authors contributed equally to this work.

Received 30 March 2012; revised 30 July 2012; accepted 29 August 2012
} 
HCC. ${ }^{17,18}$ Mitochondria are involved in cancer development and progression. ${ }^{19,20}$ Mitochondria are dynamic organelles undergoing continuous fission and fusion to form a reticulum structure that is considered to be an important determinant of mitochondria function. ${ }^{21,22}$ In mammalian cells, mitochondrial fusion and fission rely on multiple proteins, including fusion modulating mitofusin 1 (Mfn1), mitofusin 2 (Mfn2), and optic atrophy 1 (OPA1), as well as fission-related Drp1 (dynamin-related protein Drp1) and Fis1 (fission 1). ${ }^{23}$ Mitochondrial dynamics have an important role in the process of apoptosis. Many apoptotic stimuli can elicit mitochondrial fragmentation during the early stages of apoptosis, and in microscopy studies this is characterized by the formation of abundant smaller and more-rounded mitochondria. Inhibition of mitochondrial fragmentation not only preserves the mitochondrial architecture but also prevents the release of cytochrome $c$ and subsequent apoptotic steps. The mitochondria are an attractive target for cancer therapeutic development. ${ }^{24}$

This study aims to further clarify the mode of function by which sorafenib acts. We provide evidence to show that sorafenib induces apoptosis in HCC cells through disruption of functionally modified mitochondria rather than by inhibition of the Ras-Raf or phosphoinositide 3-kinase-protein kinase B (PI3K-Akt) signal pathways. Our data indicate that mitochondria fusion protein OPAl expression level is critical to determining the sensitivity of HCC to sorafenib-induced apoptosis.

\section{MATERIALS AND METHODS Ethics Statement}

With the approval of the University of Florida Gainesville Health Science Center Institutional Review Board (IRB-01), archived frozen liver cancer tissues and paired non-tumor liver tissues were used in this study. No donor organs were obtained from executed prisoners or other institutionalized persons.

\section{Materials}

MitoTracker ${ }^{\mathbb{B}}$-Red CMXRos was obtained from Invitrogen (Carlsbad, CA, USA); anti- $\beta$-actin, anti-Mfn 1 monoclonal antibodies, and Hoechst 33258 were obtained from Sigma (St Louis, MO, USA); anti-Mfn2 and anti-OPA1 primary antibodies were purchased from Abcam (Cambridge, MA, USA); anti-caspase 9, anti-caspase 3 (cleaved (c)), antiphosphorylated (p)-c-Raf, anti-c-Raf, anti-K-Ras (Kirsten rat sarcoma viral oncogene homolog), anti-Akt, and anti-p473Akt primary antibodies were obtained from Cell Signaling Technology (Beverly, MA, USA); anti-voltage-dependent anion-selective channel 1 (VDAC1) primary antibody, goat anti-rabbit, and goat anti-mouse horseradish peroxidase (HRP)-conjugated secondary antibodies were purchased from Santa Cruz Biotechnology (Santa Cruz, CA, USA); and anti-cytochrome $c$ monoclonal antibody was obtained from BD Bioscience (San Diego, CA, USA). Sorafenib was obtained from Selleck Chemicals (Houston, TX, USA); CellTiter ${ }^{\circledR} 96$ Aqueous Non-Radioactive Cell Proliferation Assay (3-(4,5-dimethylthiazol-2-yl)-5-(3-carboxymethoxyphenyl)2-(4-sulfophenyl)-2H-tetrazolium (MTS)) was purchased from Promega (Madison, WI, USA).

\section{Cell Culture and Reagents}

Human HCC cell lines (Huh7 and LH86) were grown in Dulbecco's modified Eagle's medium (DMEM) with $10 \%$ fetal bovine serum (Sigma) and antibiotics $(100 \mathrm{U} / \mathrm{ml}$ penicillin and $100 \mu \mathrm{g} / \mathrm{ml}$ streptomycin) at $37^{\circ} \mathrm{C}$ in $5 \% \mathrm{CO}_{2}$. Normal primary human hepatocytes were obtained from CellzDirect (Austin, TX, USA). The cells were cultured in the DMEM/F12 (1:1) culture medium.

\section{Cell Viability Assay (MTS Assay)}

Cell viability assays were performed as described previously. ${ }^{25}$ Briefly, cells were untreated or treated with sorafenib, and cell viability was determined using the CellTiter ${ }^{\mathbb{E}} 96$ Aqueous One Solution Cell proliferation Assay kit (Promega) according to the supplier's protocol.

\section{Western Blotting Analysis}

Western blotting was performed as described previously. ${ }^{26}$ Cells were treated and harvested. The cell pellets were resuspended in lysis buffer containing Nonidet P-40. After centrifugation, the supernatant was transferred to a new tube and the protein concentration was determined. Equivalent amount of samples were subjected to sodium dodecyl sulfate (SDS)-polyacrylamide gel electrophoresis on 12\% gels. Proteins were transferred to nitrocellulose membranes and probed with the indicated primary antibodies, followed by the appropriate secondary antibodies. Immunoreactive bands were detected using enhanced chemiluminescence (Pierce, Rockford, IL, USA). The molecular sizes of the proteins detected were determined by comparison with prestained protein markers (Bio-Rad, Hercules, CA, USA).

\section{Detection of Apoptosis by Hoechst 33258 Staining}

Cells were plated at a density of $5 \times 10^{4}$ cells per $\mathrm{ml}$ in sixwell plates and treated with sorafenib. After treatment for desired intervals, cells were stained with Hoechst 33258 for $15 \mathrm{~min}$ at room temperature in the dark. Cells were then analyzed using fluorescence microscope. For quantitative analysis, apoptotic cell death ratio was assessed by calculating the number of apoptotic cells with condensed nuclei in 6-8 randomly selected areas.

\section{Immunofluorescence Staining}

To assess the modification of mitochondrial morphology, cells grown on glass coverslips were treated with sorafenib for different intervals. MitoTracker ${ }^{\mathbb{B}}$-Red probe was directly added into the culture medium and the culture plate was incubated for $15 \mathrm{~min}$ at $37^{\circ} \mathrm{C}$ in the dark. Cells were washed with phosphate-buffered saline (PBS), fixed with 3.7\% 
paraformaldehyde at $37^{\circ} \mathrm{C}$ for $15 \mathrm{~min}$, and then quenched with $50 \mathrm{mM} \mathrm{NH}_{4} \mathrm{Cl}$. The coverslips were then extensively washed and mounted in Mowiol reagent. Fluorescent images were obtained using an Olympus fluorescence microscope.

\section{Isolation of Mouse Mitochondria and Measurement of Cytochrome C Release}

Mouse liver mitochondria were isolated as described previously, ${ }^{27}$ and the protein content of isolated mitochondria was determined by the micro-biuret method using BSA as a standard. Equal mitochondrial fractions were treated with different concentrations of sorafenib. The samples were then centrifuged and cytochrome $c$ released into the supernatant was detected by western blotting.

\section{SiRNA-Mediated Knockdown}

The siRNA knockdown experiments were performed as described previously. ${ }^{28}$ Smart-pool pre-designed siRNA duplexes targeted against human OPA1 mRNA were obtained from Cell Signaling Technology. Cells were transfected with $100 \mathrm{nM}$ siRNA duplex mixtures for $24 \mathrm{~h}$ in the presence of Lipofectamine RNAiMax (Invitrogen) according to the manufacturer's instructions. A nonspecific random siRNA (Cell Signaling Biotechnology) was also transfected at the same concentration as the control.

\section{Detection of Apoptosis by DNA Ladder Assay}

A DNA ladder assay was performed as described previously with modification. ${ }^{29}$ Briefly, cells treated under different conditions were harvested and washed with $1 \times$ PBS. Cell pellets were resuspended with lysis buffer. The samples were centrifuged and supernatants were transferred to new tubes. SDS and RNase A were added to the supernatants, and the mixture was incubated at $56^{\circ} \mathrm{C}$ for $2 \mathrm{~h}$. Proteinase $\mathrm{K}$ was added and the samples were incubated at $37^{\circ} \mathrm{C}$ for $2 \mathrm{~h}$. DNA was precipitated with the addition of $10 \mathrm{M}$ ammonium acetate and ethanol, washed once with $70 \%$ ethanol, and then dissolved in water and separated by electrophoresis in $1 \%$ agarose gel.

\section{Subcellular Fractionation}

This assay was performed as described previously. ${ }^{30}$ Cells were harvested and resuspended in three volumes of hypotonic buffer. After gentle homogenization with a dounce homogenizer, cell lysates were centrifuged to remove unbroken cells and nuclei. The post-nuclear supernatant was centrifuged to pellet the mitochondriaenriched heavy membrane fraction. The supernatant was further centrifuged to obtain the cytosolic fraction. A 30- $\mu \mathrm{l}$ aliquot cytosolic fraction was analyzed by western blotting with anti-cytochrome $c$ monoclonal antibody.

\section{Tumor Growth Inhibition Assay}

The 8-week-old SCID mice managed humanely according to the criteria outlined in the 'Guide for the Care and Use of
Laboratory Animals' (NIH publication 86-23, revised 1985) were divided into two groups. Each mouse (4 per group) was inoculated subcutaneously with $5 \times 10^{6}$ cells resuspended in $100 \mu \mathrm{l}$ PBS in the right flank. Treatment with sorafenib was initiated when tumors were palpable (day 7 after inoculation) by daily per oral administration of sorafenib $(100 \mathrm{mg} / \mathrm{kg}$ body weight) or vehicle only for a total of 7 days. At the end point of the experiment, mice were euthanized following the University of Florida IACUC-approved protocols. Each tumor was excised, weighed, and fixed in formalin. The effect of drug treatment on tumor growth was analyzed statistically with individual group comparison and evaluated with the Student's paired $t$-test by SPSS 15.0.

\section{RESULTS}

\section{Sorafenib Induces Apoptosis in HCC Cells}

To investigate the apoptotic effects of sorafenib on HCC cells, we incubated Huh7 and LH86 cells with sorafenib. MTS assays demonstrated that sorafenib induced significant concentration-dependent cell growth inhibition in both cell lines (Figure 1a). Nuclear condensation assays showed that sorafenib treatment induced significant apoptotic nuclear condensation in both cell types (Figures $1 \mathrm{~b}$ and $\mathrm{c}$ ). Consistent with the results shown in Figure 1b, DNA ladder assays also demonstrated that sorafenib treatment resulted in apoptosis in a concentration-dependent manner in both HCC cell lines (Figure 1d). To further evaluate sorafenib-induced apoptosis in HCC cells, caspase activation was assessed by western blotting analysis. Our data indicated that treatment with sorafenib triggered proteolytic processing of caspase 9 and caspase 3 (Figure 1e). Taken together, these results suggest that sorafenib induces apoptosis in HCC cell lines and the proteolytic activation of caspase 9 and caspase 3 indicates that sorafenib may trigger mitochondria-mediated apoptosis.

\section{Effects of Sorafenib on Ras-Raf and PI3K-Akt Antiapoptotic Signal Pathways}

It has been reported that sorafenib induces apoptosis by inactivation of the Ras-Raf and Akt survival pathways in nonHCC cells. ${ }^{31,32}$ To gain further insight into the downstream apoptotic effects of sorafenib on HCC, we analyzed the expression of K-Ras, c-Raf, p-c-Raf, p473-Akt, and Akt in response to sorafenib treatment. We found that treatment with sorafenib induced upregulation of p-c-Raf and p473Akt in a time-dependent manner. Expression levels of total K-Ras, Akt, and c-Raf were not significantly affected (Figures $2 \mathrm{a}$ and $\mathrm{b})$. More importantly, we observed that p-c-Raf and p473-Akt were increased in sorafenib-treated cells in a concentration-dependent manner, even though apoptosis occurred as mentioned in Figure 1 (Figures $2 \mathrm{c}$ and $\mathrm{d}$ ). These results suggest that treatment with sorafenib increases activation of the Raf and Akt signaling pathways in the HCC cells, and that the effect of sorafenib may be cell type dependent or sorafenib targets other pathways to induce apoptosis in HCC cells. 


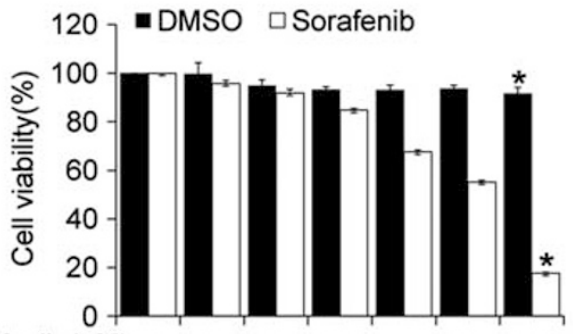

Sorafenib $(\mu \mathrm{M})$

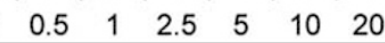

Huh7

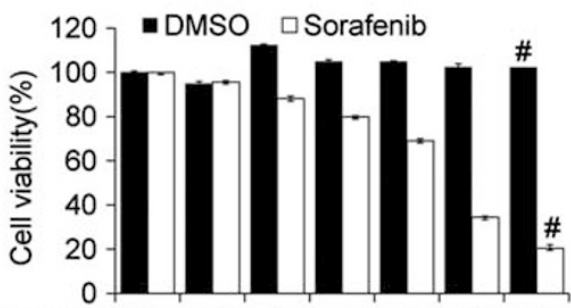

Sorafenib $(\mu \mathrm{M})$

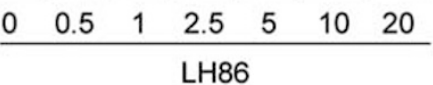

LH86

b

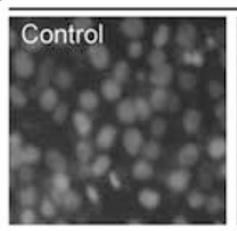

Huh7
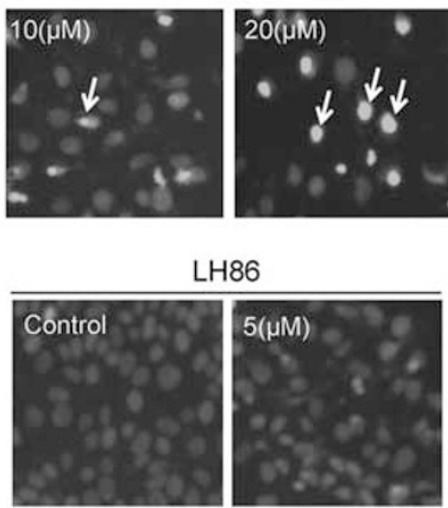

LH86

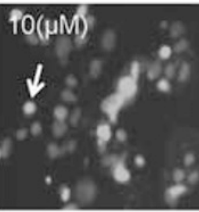

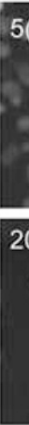

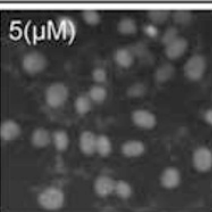

$20(\mu \mathrm{M})$
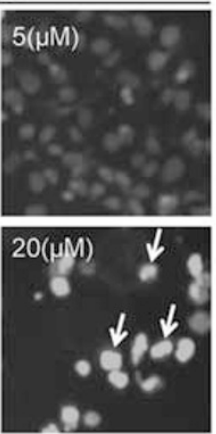
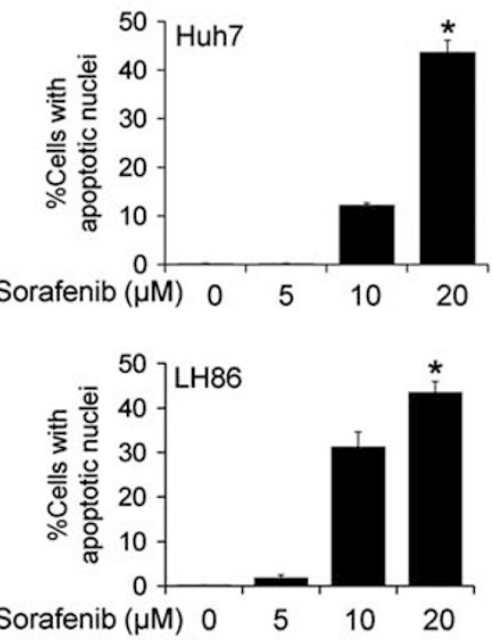

d

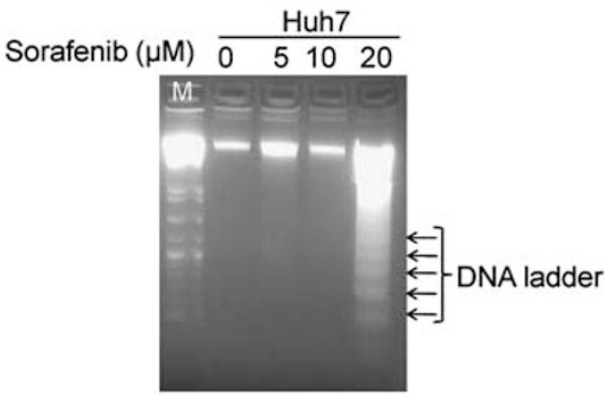

e

Time (h) $\begin{array}{lllllllll}0 & 6 & 12 & 24 & & 0 & 6 & 12 & 24\end{array}$

Cleaved caspase 9

Cleaved caspase 3

$\beta$-actin
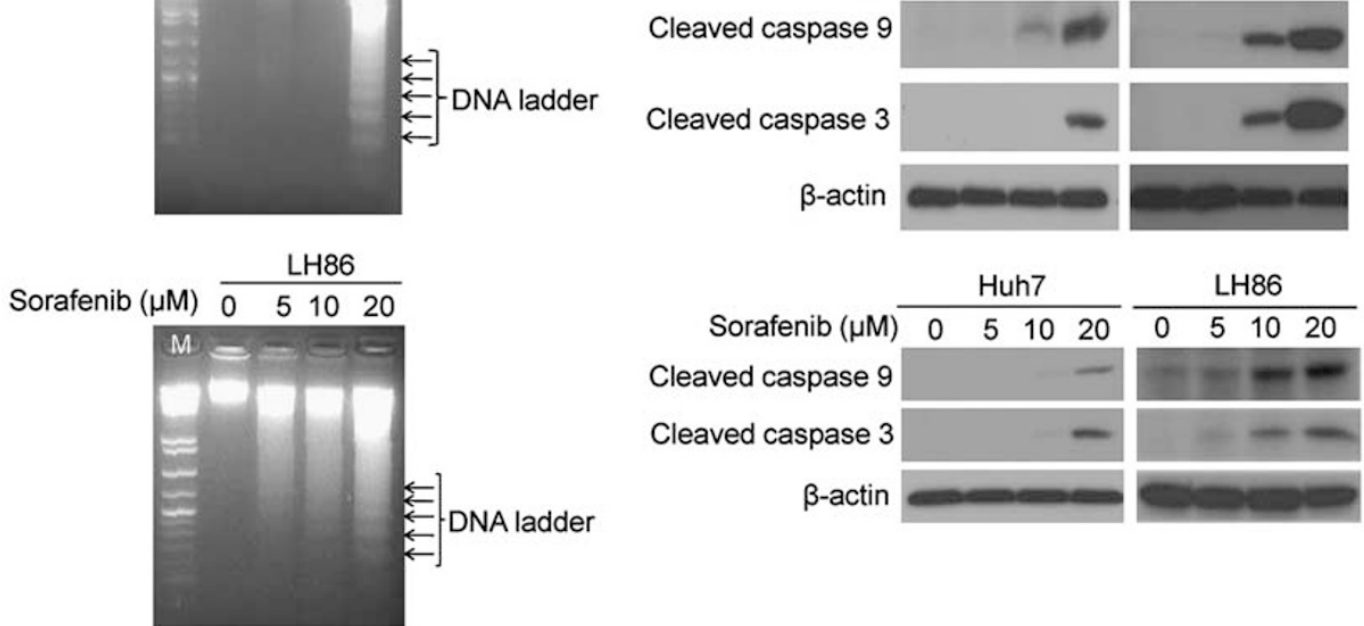

Figure 1 Sorafenib induces apoptosis in hepatocellular carcinoma (HCC) cells. (a) Huh7 and LH86 cells were treated with sorafenib as indicated. The 3-(4,5-dimethylthiazol-2-yl)-5-(3-carboxymethoxyphenyl)-2-(4-sulfophenyl)-2H-tetrazolium (MTS) assay was performed to detect cell viability ${ }^{*} P<0.05$, $\left.{ }^{\#} P<0.05\right)$. (b) Cells were treated with sorafenib as indicated. Nuclear condensation was assessed by Hoechst staining to show apoptotic nuclei as described in 'Materials and Methods'. (c) Cells were treated with sorafenib and nuclear condensation was assessed by 33258 staining. The apoptosis ratio was analyzed by counting cells with apoptotic nuclei under a fluorescent microscope (representative apoptotic cells were marked with white arrows, ${ }^{*} P<0.05$ ). (d) Cells were treated with sorafenib and DNA ladder assay was carried out to show DNA fragmentation (M: $1 \mathrm{~kb}$ plus DNA marker). (e) HCC cells were treated with sorafenib $(20 \mu \mathrm{M})$ for different times (top panel) or were treated with various concentrations of sorafenib for $24 \mathrm{~h}$ (bottom panel). Cell lysates were prepared and subjected to western blotting to examine cleaved caspase 9 and caspase 3 protein levels with specific antibodies. $\beta$-Actin was used as an equal protein loading control. 

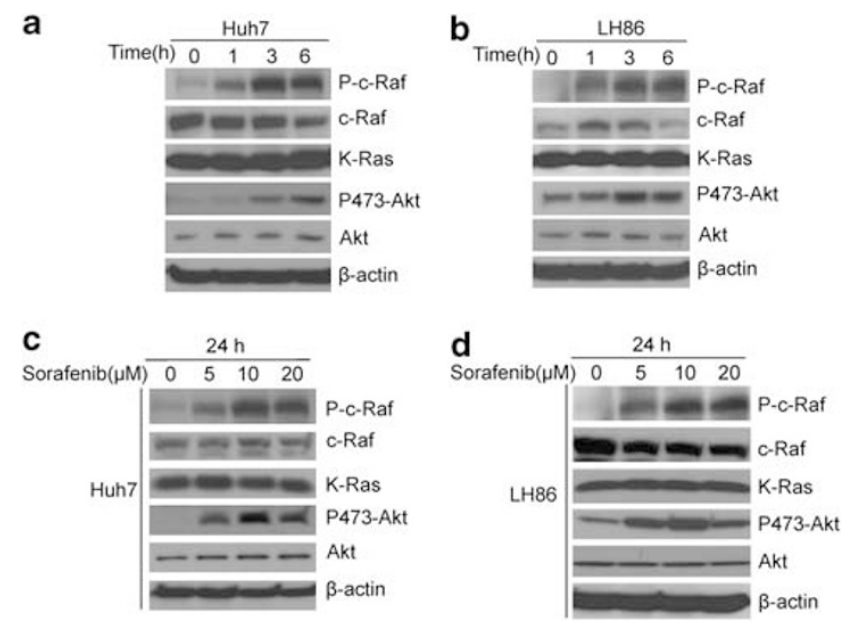

Figure 2 Effects of sorafenib on Ras-Raf and phosphoinositide 3-kinaseprotein kinase B (PI3K-Akt) signal pathways. (a) Huh7 and (b) LH86 cells were treated with sorafenib as indicated. Cell lysates were prepared and subjected to western blotting to detect phosphorylated Raf ( $p$-c-Raf), cleaved (c)-Raf, K-Ras (Kirsten rat sarcoma viral oncogene homolog), p473-Akt, and Akt with specific antibodies, respectively. $\beta$-Actin was used as an equal protein loading control. (c) Huh7 and (d) LH86 cells were treated with various concentrations of sorafenib for $24 \mathrm{~h}$. Cell lysates were prepared and subjected to western blotting to detect p-c-Raf, c-Raf, K-Ras, p473-Akt, and Akt with specific antibodies, respectively. $\beta$-Actin was used as an equal protein loading control.

\section{Sorafenib Induces Mitochondrial Fragmentation in HCC Cells}

To examine if sorafenib can induce mitochondrial dynamic changes, cells were treated with sorafenib and then stained with membrane potential-dependent MitoTracker ${ }^{\mathbb{B}}$-Red probes to show mitochondrial morphology and membrane potential. The mitochondrial network was severely damaged after sorafenib treatment, which was reflected by the presence of smaller, more-rounded mitochondria (from $15 \mathrm{~min}$ ) and membrane potential loss (at $60 \mathrm{~min}$ ) (Figures $3 \mathrm{a}$ and b). The number of cells with fragmented mitochondria increased upon additional incubation time (Figures $3 \mathrm{c}$ and $\mathrm{d}$ ). These results suggest that sorafenib induces cellular mitochondria fragmentation in a time-dependent manner in HCC cells.

\section{Sorafenib Induces Mitochondria Cytochrome C Release}

To provide further evidence that sorafenib induces apoptosis through targeting mitochondria dynamics, Huh7 cells were treated with various concentrations of sorafenib. We observed that sorafenib induces cytochrome $c$ release into the cytosol in cultured HCC cells (Figures 4a and b). Furthermore, to eliminate the possibility that sorafenib may be indirectly interacting with other cellular mechanisms or pathways, purified mitochondria were studied on incubation with sorafenib. Our data showed that sorafenib could directly induce cytochrome $c$ release from mitochondria purified from HCC cells (Figure 4c). We found that sorafenib triggered cytochrome $c$ release from mitochondria isolated from normal mouse liver tissues in a concentration- and timedependent manner (Figures $4 \mathrm{~d}$ and e). These data suggest that not only does sorafenib abrogate mitochondrial remodeling in HCC cells but also that there is sufficient mitochondrial disruption to release cytochrome $c$ into the cytoplasm to induce apoptosis. The data also provide evidence that sorafenib directly interacts with the mitochondria and does not act by an intermediate mechanism.

\section{Sorafenib Deregulates Expression of Mitochondrial Fusion Protein OPA1}

Dynamic control of mitochondrial structure is maintained by a number of mitochondria-shaping proteins that include both fusion (Mfn1, Mfn2, and OPA1) and fission members. OPA1 is a major organizer of the mitochondrial inner membrane, maintains mitochondria DNA stability and replication, and is involved in the sequestration of cytochrome $c$. It is reasonable to hypothesize that OPA1 may be a target for mitochondrial apoptotic effectors. ${ }^{33}$ To determine the mechanisms underlying sorafenib-induced mitochondrial fragmentation, Huh7 and LH86 cells were treated with sorafenib for $0-60 \mathrm{~min}$. We found that the expression of mitochondria fusion-related protein OPA1 was decreased, whereas the other two members, Mfn1 and Mfn2, were largely unchanged in both cell types (Figure 5a). Further experiments showed that, upon treatment with sorafenib over $24 \mathrm{~h}$, OPA1 down regulation was more profound, with additional downregulation of Mfn1 and Mfn2 (Figure 5b). As the loss of OPA1 expression is a common consequence of sorafenib treatment in both HCC cell lines, we decided to investigate the role of OPA1 expression further to determine if OPA1 regulation by sorafenib is involved in apoptosis of HCC cells. We observed that OPA1 knockdown (Figure 5c) could significantly sensitize Huh7 cells to sorafenib-induced apoptosis (Figure 5d and Supplementary Figure S1). These data suggest that OPA1 is a target of sorafenib, and that by targeted knockdown of OPA1, HCC cells can be further sensitized to apoptosis induced by sorafenib.

\section{Sorafenib has no Apoptotic Toxicity to Normal Human Liver Cells}

We have shown that sorafenib induced apoptosis in HCC cell lines by targeting OPA1. OPA1 has an important role in mitochondrial remodeling and stability; therefore, to determine if sorafenib treatment is toxic to normal human primary liver cells (hepatocytes), both hepatocytes and HCC cells were treated with sorafenib. We found that sorafenib had no detectable apoptotic effects on hepatocytes, whereas sorafenib treatment did induce apoptosis in HCC cells that served as a positive control (Figure 6a). To assess whether sorafenib can induce mitochondria fragmentation in hepatocytes, cells were untreated or treated with sorafenib. We found that sorafenib could not induce apparent mitochondrial fragmentation in hepatocytes, while it did in positive control HCC cells (Figure 6b). To understand why 
a
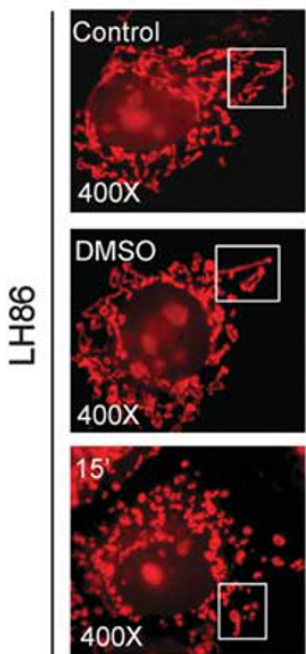

400X: magnification
Zoom in
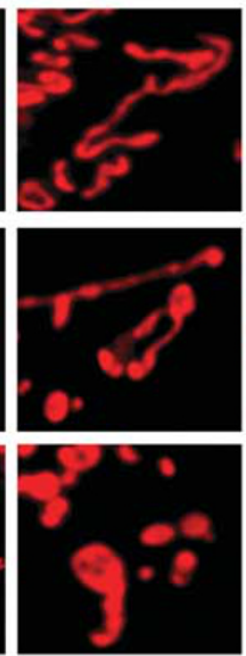
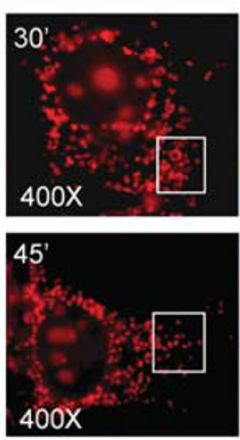

60

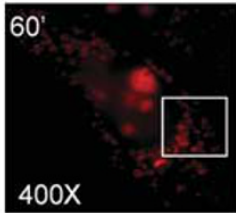

MitoTracker stained Mitochondria (Red)
Zoom in
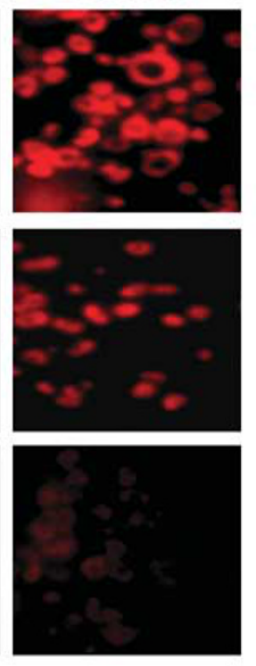

b
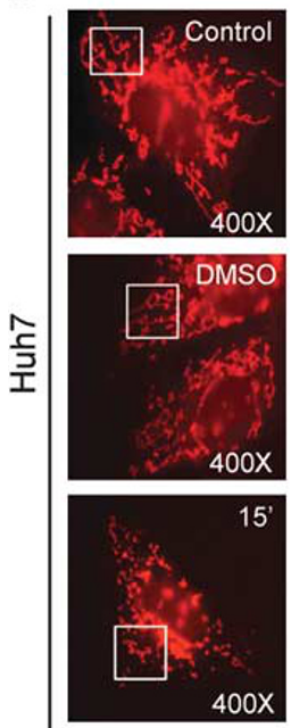

$400 x$
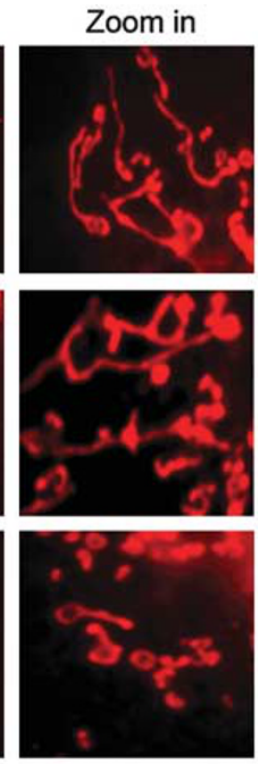

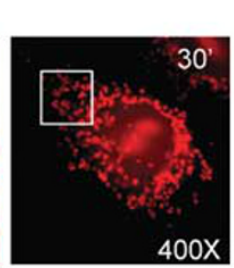

$45^{\prime}$
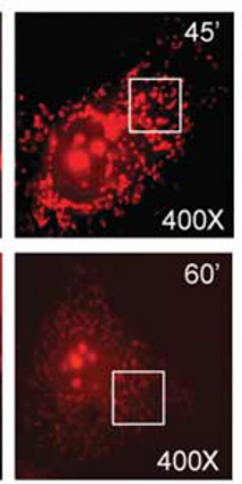
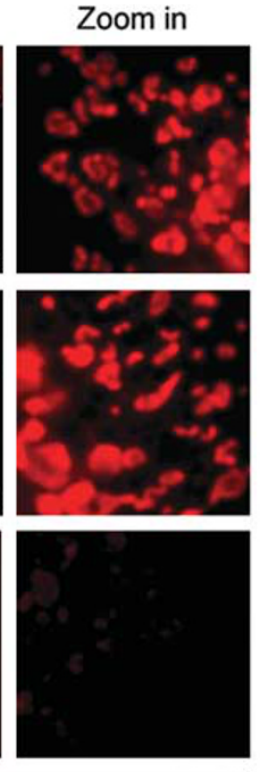

4.00X: magnification

MitoTracker stained Mitochondria (Red)

C

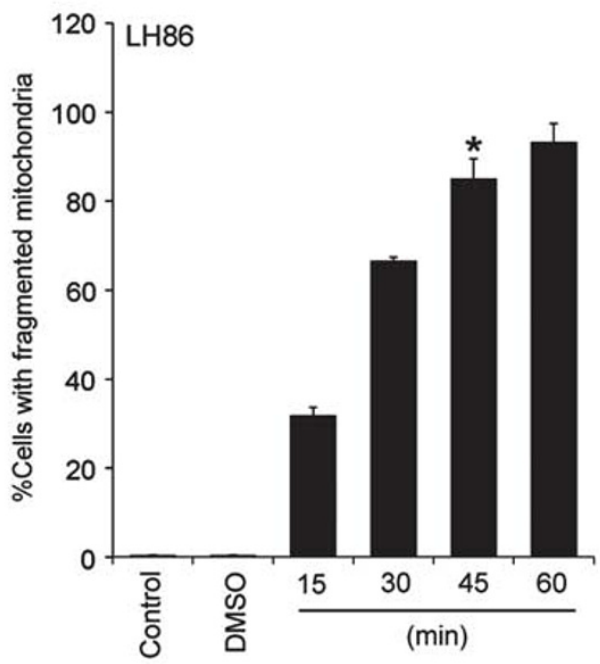

d

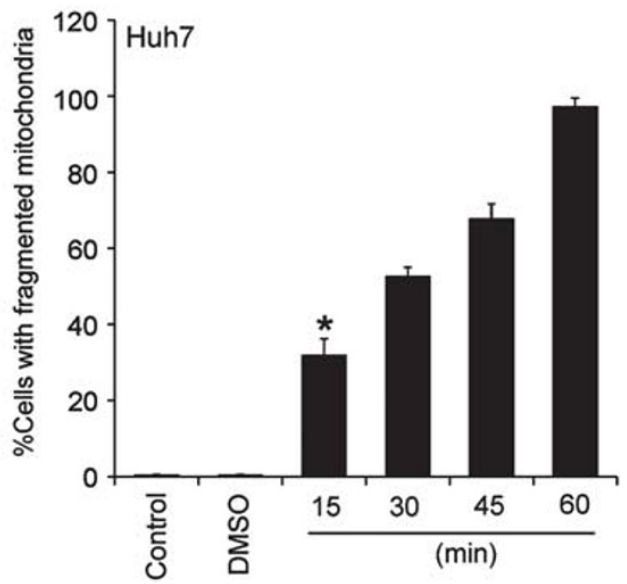

Figure 3 Sorafenib induces mitochondrial fragmentation in hepatocellular carcinoma (HCC) cells. (a) LH86 and (b) Huh7 cells were treated with sorafenib and were stained with mitochondria membrane-dependent MitoTracker ${ }^{\mathbb{E}}$-Red probe. Then, mitochondria morphology was observed under fluorescence microscope (Red) (areas marked with white boxes were enlarged (zoom-in)). (c) LH86 and (d) Huh7 cells were treated with sorafenib $(20 \mu \mathrm{M})$ for indicated times and the number of cells with fragmented mitochondria was counted $\left({ }^{*} P<0.05\right)$.

the mitochondria of hepatocytes are resistant to sorafenib, we compared mitochondrial fusion protein expression between hepatocytes and HCC cells. Hepatocytes express markedly higher levels of OPA1, Mfn1, and Mfn2 than HCC cells (Figure 6c). To further assess the roles of mitochondria fusion-related protein in hepatocytes, cells were treated with sorafenib for a short time course $(0-60 \mathrm{~min})$ and a longer time course for $24 \mathrm{~h}$. We found that mitochondria fusion proteins OPA1, Mfn1, and Mfn2 were not significantly affected by sorafenib treatment in hepatocytes (Figures 6d and e). These results suggest that hepatocytes are resistant to sorafenib and that the mitochondrial dynamic processes in hepatocytes are not affected by sorafenib treatment; the data also show that HCC cells with lower expression of OPA1, Mfn1, and Mfn2 are less resistant to apoptotic induction by sorafenib.

\section{Sorafenib Exerts Tumor Growth Inhibition In Vivo}

To investigate whether the antitumor effects of sorafenib in vivo are mediated by mitochondria fragmentation, HCC xenograft tumor models were established in SCID mice by injection of HCC cells. Analysis of HCC xenograft tumors 
a

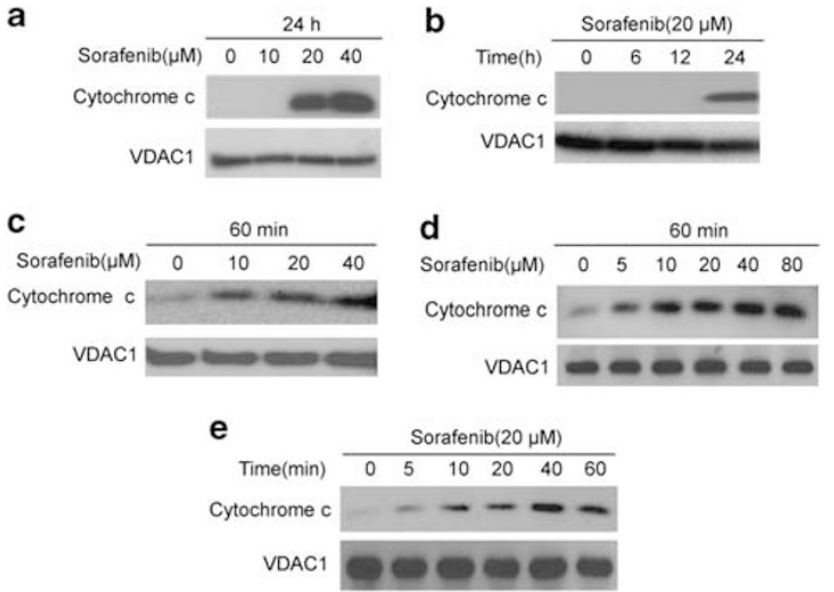

Figure 4 Sorafenib induces cytochrome $c$ release. (a) Huh7 cells treated with different concentrations of sorafenib or (b) with a various time periods were harvested and mitochondria were purified by subcellular fractionation. Cytochrome $c$ in the supernatant was detected with anticytochrome $c$ monoclonal antibody. Voltage-dependent anion-selective channel 1 (VDAC1) from total mitochondria was used as a control for equal protein loading. (c) Isolated mitochondria from hepatocellular carcinoma (HCC) cells were incubated with sorafenib. Cytochrome $c$ release was assessed by western blotting with specific cytochrome $c$ antibody. VDAC1 was set up for equal protein loading control. (d) Purified mouse liver mitochondria were incubated with different concentrations of sorafenib and cytochrome $c$ release was measured by western blotting. VDAC1 was used as a control for equal protein loading. (e) Mitochondria were prepared from mouse liver tissue. The same amount of mitochondria was treated with sorafenib for time periods as indicated. Cytochrome $c$ levels were detected by western blotting with cytochrome $c$ antibody. VDAC1 was used as a control for equal protein loading.

from the control buffer- and sorafenib-treated mice revealed that sorafenib administration effectively inhibits HCC tumor growth in vivo (Figure 7a). There was a detectable difference in tumor mass with at least a 10 -fold decrease in sorafenibtreated mice when compared with untreated control mice (Figure 7b). Moreover, tumor tissues from untreated or treated groups were analyzed to examine OPA1, Mfn1, and Mfn2 expression levels. Our results indicated that sorafenib treatment significantly reduced the expression of OPA1 as well as Mfn1 and Mfn2 in xenograft HCC tumors (Figure 7c). Similar to the in vitro findings, these results suggest that sorafenib inhibits HCC tumor growth in vivo by targeting mitochondria dynamic-related proteins.

\section{OPA1 Expression in Human Normal Liver Tissues and Malignant Liver Tissues}

To establish the clinical relevance of these data, paired adjacent normal liver tissue and malignant HCC liver tissue from patients were collected and the expression of mitochondria fusion-related proteins, including OPA1, Mfn1, and Mfn2, were analyzed by western blotting. Matched comparisons with adjacent normal liver tissues showed that there was consistent downregulation of OPAl protein expression in 12 out of 30 randomly selected liver tumor
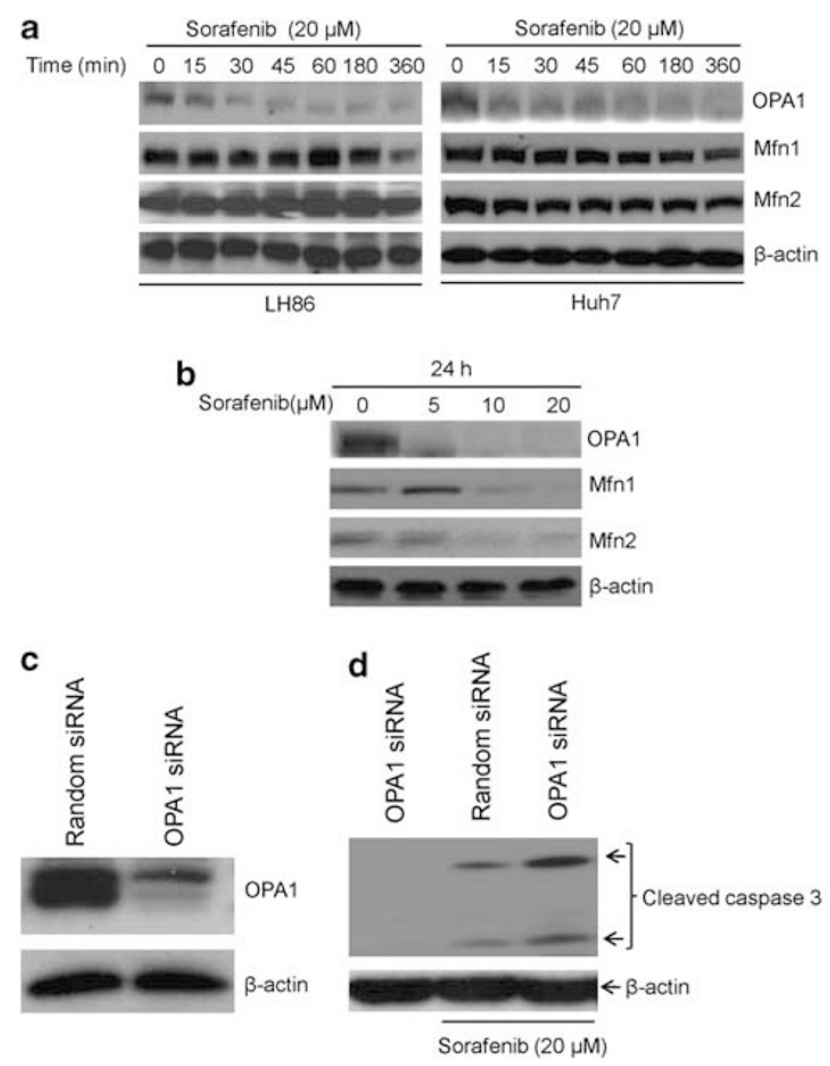

Figure 5 Sorafenib deregulates the expression of mitochondrial fusion protein optic atrophy 1 (OPA1). (a) Hepatocellular carcinoma (HCC) cells were treated with sorafenib for up to $360 \mathrm{~min}$ and harvested. Cell lysates were prepared and subjected to western blotting to examine protein levels of OPA1, mitofusin 1 (Mfn1), and mitofusin 2 (Mfn2) with specific antibodies, respectively. $\beta$-Actin was used as an equal protein loading control. (b) Huh7 cells were treated with various concentrations of sorafenib for $24 \mathrm{~h}$ and cell lysates were prepared and subjected to western blotting to detect OPA1, Mfn1, and Mfn2 expression levels with specific antibodies, respectively. $\beta$-Actin was used as an equal protein loading control. (c) Huh7 cells transfected with synthesized random control small interfering RNA (siRNA) and specific OPA1 siRNA were treated with sorafenib. Cells were harvested and subjected to western blotting analysis. OPA1 expression was detected with specific antibody. (d) Cleaved caspase 3 expression level was examined with specific polyclonal antibodies. $\beta$-Actin was served as an equal protein loading control.

tissues (only representative data were shown) (Figure 8a). However, as we know that more than $90 \%$ of HCC arise from cirrhotic background, it is likely that the comparison was made between cirrhotic liver tissues and HCC tissues. In our study, further tissue analysis revealed that the OPA1 expression levels in matched HCC tissues (HCC) are lower by $50 \%$ than those in normal (non-cirrhotic) liver tissues (NL), and the difference between matched cirrhotic tissues (Cirrhotic) and HCC tissues (HCC) is minimal (Figure $8 \mathrm{~b}$ ).

\section{DISCUSSION}

Approved by the FDA to treat unresectable HCC, sorafenib is currently the main drug used in the treatment of patients with advanced HCC. ${ }^{34-36}$ Although oral administration of 

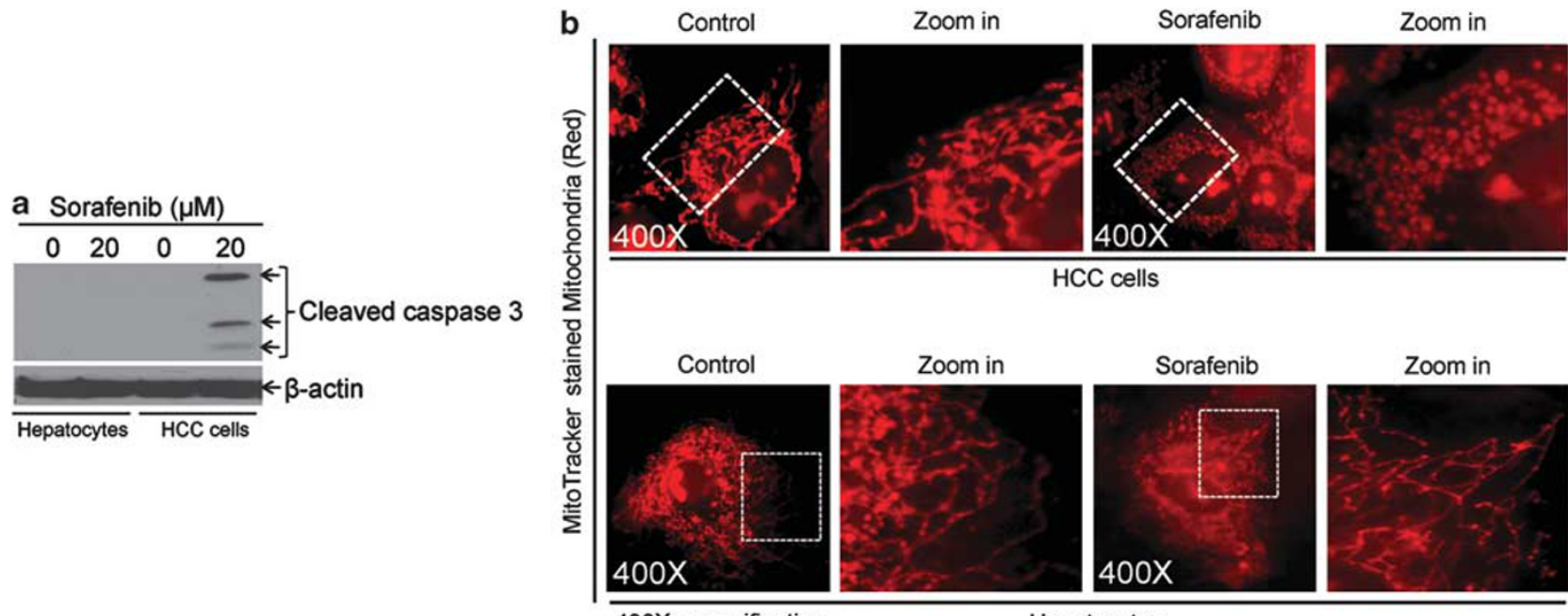

400X: magnification

Hepatocytes
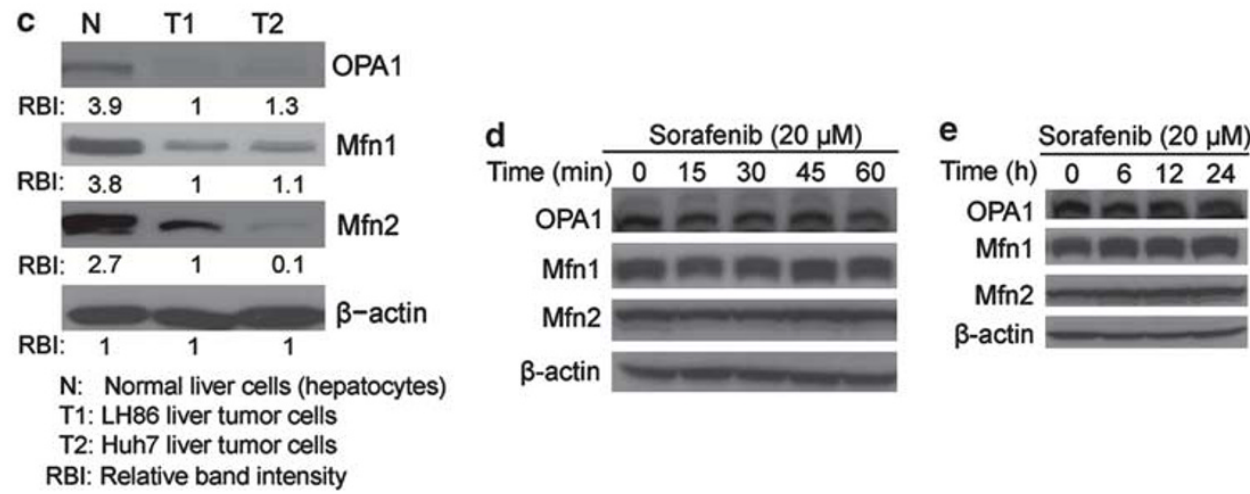

Figure 6 Sorafenib has no apoptotic toxicity to normal primary liver cells. (a) Normal human hepatocyte cultures or LH86 cells (positive control) were treated with sorafenib. Caspase 3 activation was measured by detecting the cleaved bands through western blotting. (b) Hepatocellular carcinoma (HCC) or normal hepatocytes were treated with sorafenib. MitoTracker ${ }^{\mathbb{B}}$-Red probe was added into the culture medium. Mitochondria morphology was observed by fluorescence microscope (Red). White boxes demarcate areas further magnified (zoom-in). (c) Cell lysates from normal hepatocytes, LH86, and Huh7 were prepared and subjected to western blotting. Optic atrophy 1 (OPA1), mitofusin 1 (Mfn1), and mitofusin 2 (Mfn2) expression levels were assessed with specific antibodies, respectively. $\beta$-Actin was used as an equal protein loading control. Relative band intensities (RBIs) were analyzed by the Image J software. Normal hepatocytes were treated with sorafenib (d) for a short time course or (e) a longer time course. Cell lysates were prepared and subjected to western blotting. OPA1, Mfn1, and Mfn2 expression levels were assessed with specific antibodies, respectively. $\beta$-Actin was used as an equal protein loading control.

sorafenib has been shown to improve overall survival from a median of 7.9 to 10.7 months in patients with advanced liver cancer, the efficacy and adverse reactions differ in individuals. Understanding the underlying molecular mechanisms will enable the identification of HCC patients who will benefit from a sorafenib-based treatment. In this study, we find that sorafenib could induce apoptosis by targeting mitochondria dynamics rather than inhibiting the Raf and Akt survival signal pathways. In addition, deregulation of mitochondrial fusion protein OPAl has a key role in the induction of apoptosis in HCC cells and inhibition of HCC xenograft tumor growth in vivo.

Sorafenib, commonly described as a Raf- 1 and multikinase inhibitor, has been shown to be able to interfere with the RasRaf signal pathway in diverse transformed cells.,37,38 Surprisingly, here we show that sorafenib induces activation of Raf and Akt in two different HCC cell lines, suggesting that the sorafenib-induced HCC cell apoptosis may be independent of Raf and Akt pathway inhibition. Although these findings directly contrast reports that sorafenib inhibits Ras-Raf or Akt signal pathways in other cancer cell lines. ${ }^{39-41}$ Other investigators have also reported that sorafenib could induce cell death independent of Raf/MEK/extracellular signal-regulated kinase (ERK) inhibition. ${ }^{38,42,43}$ Our findings indicate that the effects of sorafenib on Raf/ERK1/2 activation are likely to be both cell type- and context-specific.

To determine the mechanism of sorafenib-induced apoptosis in treated HCC cells, we performed a series of experiments to examine the injury of mitochondria. We observed that sorafenib treatment induced mitochondria fragmentation within $15 \mathrm{~min}$, after treatment for $60 \mathrm{~min}$, mitochondria membrane potential was significantly decreased, and fragmented 

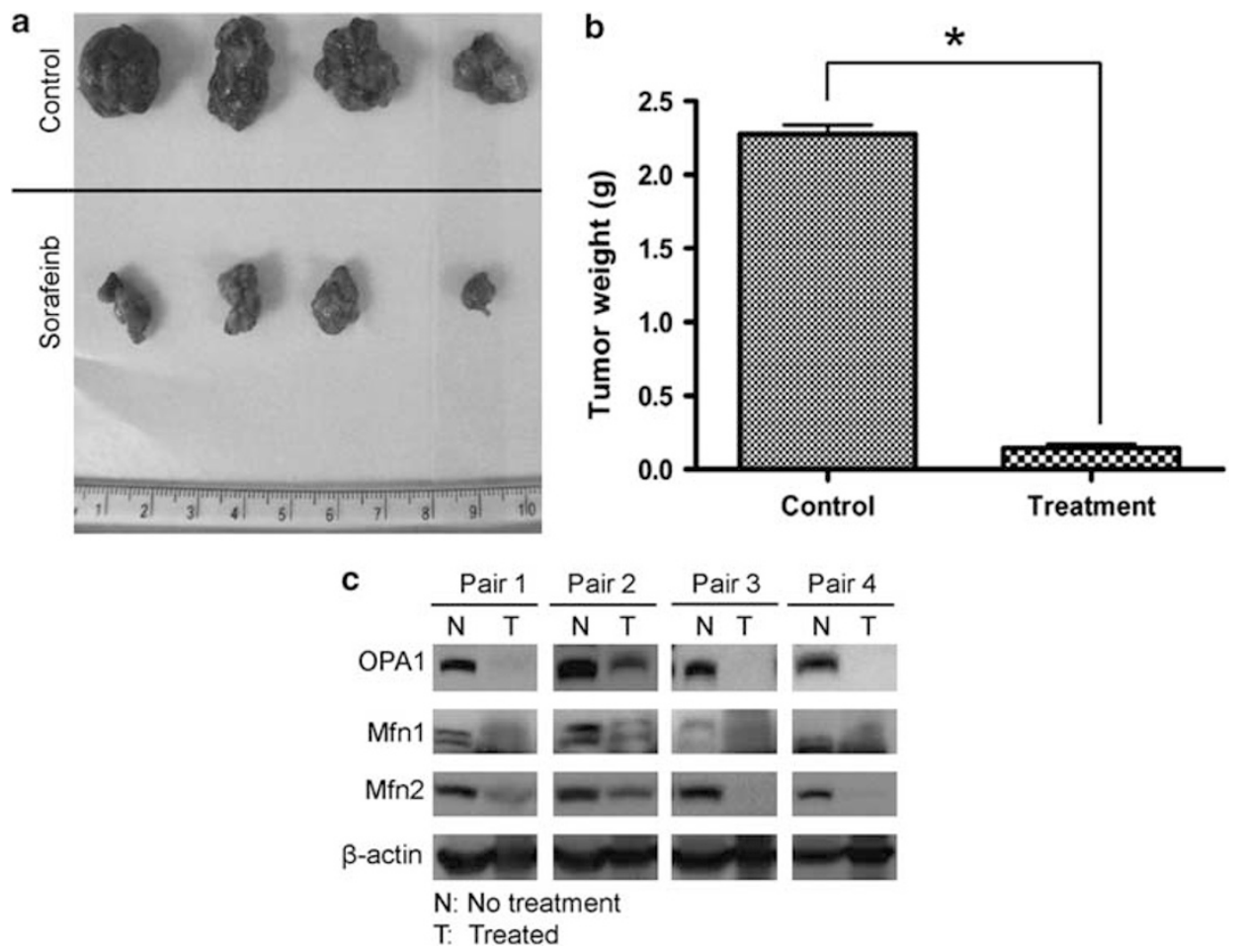

Figure 7 Sorafenib exerts tumor growth inhibition in vivo. (a) BALB/c severe combined immunodeficiency (SCID) mice were inoculated subcutaneously with Huh7 cells. The mice were treated with $100 \mathrm{mg} / \mathrm{kg}$ body weight sorafenib or vehicle orally for 7 days. At the end point of the experiment, mice were euthanized and tumors were excised. Pictures of excised tumors from xenograft mice, showing larger tumors from untreated (control) mice than those from sorafenib-treated mice. (b) Mass of tumors in nude mice from the control group and from the group treated with sorafenib were compared. Statistical analysis was performed to show inhibitory effects of sorafenib on the mass of implanted tumors in nude mice $\left({ }^{*} P<0.05\right)$. (c) Xenograft HCC tumors from untreated groups and sorafenib-treated groups were collected and lysates were prepared. Western blotting was performed to detect mitochondria fusion-related proteins optic atrophy 1 (OPA1), mitofusin 1 (Mfn1), and mitofusin 2 (Mfn2) with specific antibodies, respectively. $\beta$-Actin protein levels were assessed as an equal protein loading control. The four pairs of tissues tested were randomly selected sample pairs from untreated or sorafenib-treated tumor mice.

mitochondria were observed in up to $90 \%$ of treated cells. We also show that sorafenib induces cytochrome $c$ release when cells were treated with sorafenib at different concentrations. We provide critical evidence that sorafenib can directly induce cytochrome $c$ release when mitochondria were incubated with sorafenib in a concentration- and timedependent manner, which correlated with mitochondria fragmentation. These lines of evidence support the notion that sorafenib induces apoptosis by directly targeting mitochondria injury rather than Ras-Raf or Akt signaling pathway inhibition, at least in these evaluated HCC cells.

It is well documented that mitochondria fragmentation is regulated by mitochondria fusion- and fission-related proteins. OPA1 is a major organizer of the mitochondrial inner membrane and is important for maintaining normal mitochondria cristae structure and function and protecting cells from apoptosis by the sequestration of cytochrome $c$ release. OPA1 mutation and knockout has been shown to cause severe mitochondria morphology collapse and subsequent cell death. Also, other studies have shown that molecules such as SLP2 and PHB1 act to induce apoptosis via an OPA1-dependent pathway, which can be blocked by overexpression or functional site mutation of OPA1. ${ }^{44,45}$ In our study, to identify the molecular target that mediates mitochondria injury induced by sorafenib, we examined OPA1 expression levels in sorafenib-treated cells. Results demonstrated that sorafenib rapidly reduced the expression of mitochondrial fusion protein OPA1 within $15 \mathrm{~min}$, which coincides with mitochondria fragmentation occurrence and a delayed cytochrome $c$ release, suggesting that sorafenib promotes mitochondria fragmentation and cytochrome $c$ release by rapid downregulation of OPA1. More interestingly, we show that HCC cells can be sensitized to sorafenib-induced apoptosis by OPA1 siRNA knockdown. These data imply that OPA1 expression level is very important in determining cell sensitivity to sorafenib-induced cytotoxicity. These results are in agreement with previous reports that OPA1 downregulation induced mitochondria fragmentation and apoptosis in non-liver cancer cells. ${ }^{46}$ Our findings contribute to the idea that OPA1 is a central regulator of mitochondrial cristae structure and is essential for the maintenance of mitochondrial structure. In addition, we also observed that $\mathrm{Mfn} 1$ and Mfn2 proteins are downregulated in sorafenib-treated cells and in HCC patient tissues and correlate with OPA1 
a
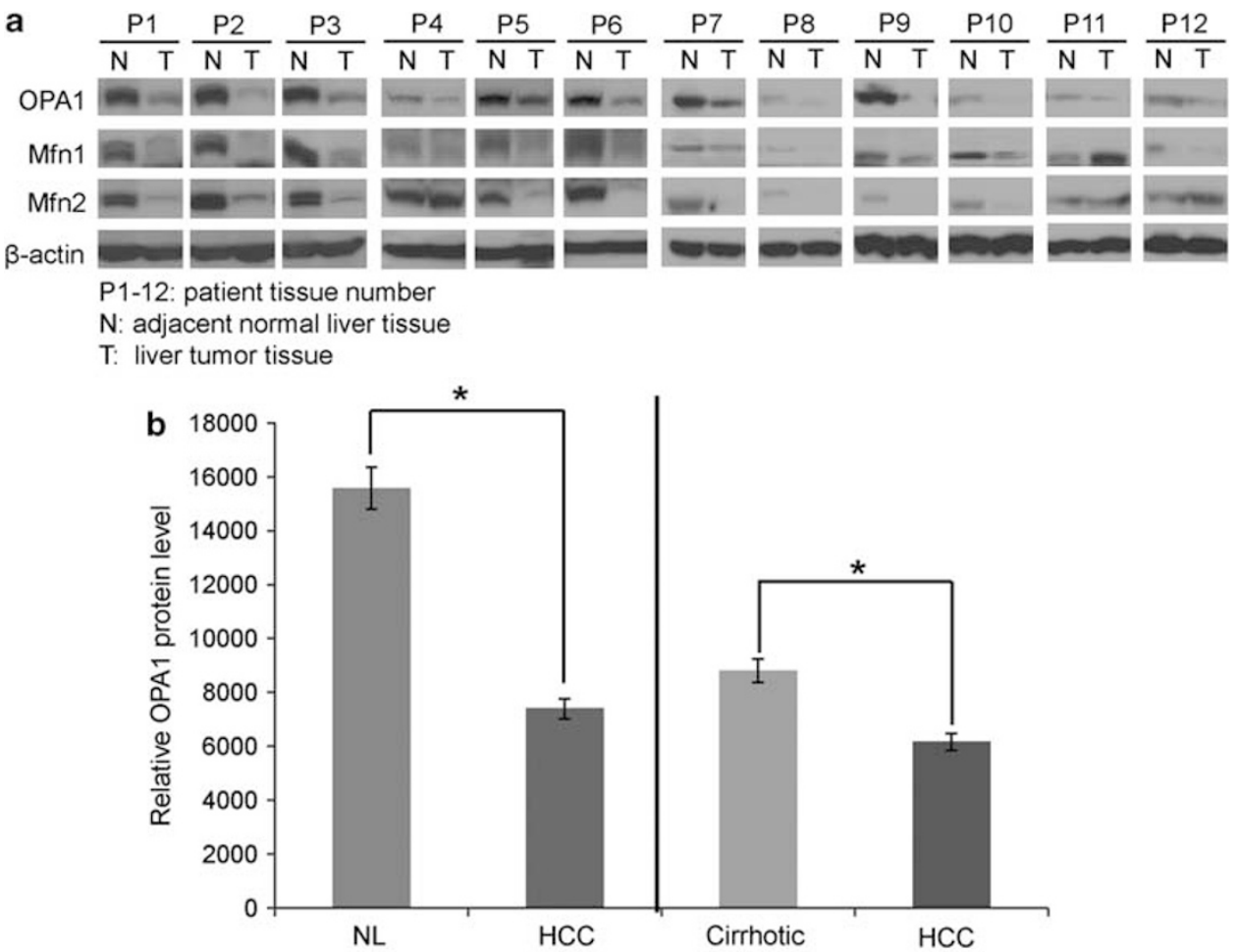

NL: normal liver tissue

HCC: liver tumor tissue

Cirrhotic: Cirrhotic liver tissue

Figure 8 Optic atrophy 1 (OPA1) expression in human normal liver tissues and liver tumor (hepatocellular carcinoma (HCC)) tissues. (a) Expression of OPA1, mitofusin 1 (Mfn1), and mitofusin 2 (Mfn2) proteins in adjacent normal tissues and malignant liver tissues. Lysates from adjacent normal liver tissues and liver tumor tissues from different patients were prepared and subjected to western blotting. OPA1, Mfn1, and Mfn2 expression levels were detected with specific antibodies. $\beta$-Actin protein levels were assessed as an equal protein loading control (P1-12: randomly selected patient number). (b) OPA1 protein level analysis in paired normal liver (NL) tissues and liver tumor (HCC) tissues, and paired cirrhotic liver tissues and liver tumor tissues (HCC). Relative band intensities (RBIs) of OPA1 were analyzed by the Image J software to show relative OPA1 protein levels in paired tissues from patients. Statistical analysis was performed to show average OPA1 expression level in different matched tissue groups $\left({ }^{*} P<0.05\right)$.

downregulation. To identify the role of the mitochondrial fusion and fission pathway, as a putative target for cancer therapy, the significance of these findings will require further investigation.

Further analysis of mitochondrial morphology in sorafenib-treated hepatocytes revealed that normal hepatocyte mitochondria are resistant to sorafenib-induced fragmentation. We then hypothesized that if OPA1 downregulation has a direct correlation with sorafenib-induced mitochondria injury, OPA1 expression in hepatocytes would not be affected by sorafenib. Our data clearly indicate that normal hepatocytes had much higher concentrations of mitochondria fusion proteins, including OPA1, Mfn1, and Mfn2 than malignant HCC cells, and OPA1 was not significantly affected by sorafenib treatment. Thus, we provide further support that mitochondria fusion protein OPA1 may play a critical role in determining the sensitivity of HCC cells to sorafenib by maintaining mitochondria dynamics and function. This is consistent with a previous study that OPA1 is a key mediator for actinomycin D-induced apoptosis in cancer cells. ${ }^{47}$
Nevertheless, further investigation is needed to clarify why mitochondria fusion proteins are not downregulated by sorafenib in normal liver cells.

OPA1 protein level stability is important for maintaining normal mitochondria cristae structure and physiological function in vivo. ${ }^{48}$ One important aim of this investigation is to define the mechanism of action of sorafenib in vivo. We show that sorafenib significantly reduced xenograft HCC tumor growth. More importantly, the levels of mitochondria fusion proteins OPA1, Mfn1, and Mfn2 were significantly decreased in tumors from sorafenib-treated mice compared with untreated mice, implying that sorafenib can also impair mitochondria dynamics and function via regulation of OPA1 in vivo. In addition, we demonstrate that for HCC clinical samples from 30 patients, when compared with the paired adjacent normal tissues, $40 \%$ of malignant tissues had lower expression of OPA1, suggesting that mitochondria dysfunction in HCC is not a rare event. Moreover, a detailed analysis of tissue samples yielded a new notion that differential OPA1 expression between HCC and matched normal (non-cirrhotic) 
liver tissues is much higher than that between cirrhotic and matched HCC tissues. Therefore, we believe that OPA1 downregulation may occur in premalignant stage (cirrhosis). Our data strongly indicate that mitochondria fusion protein levels, especially for OPA1, may serve as an independent prognostic marker for targeted administration of sorafenib to improve overall HCC survival. More extensive clinical studies are needed.

Taken together, our study suggests that sorafenib could induce apoptosis in HCC by directly targeting OPA1 to trigger mitochondrial injury. We believe that human HCC tissues have variable expression levels of mitochondria fusion-related proteins, which could be considered as a potential marker in determining administration of sorafenib to HCC patients.

Supplementary Information accompanies the paper on the Laboratory Investigation website (http://www.laboratoryinvestigation.org)

\section{ACKNOWLEDGEMENTS}

This work was financially supported, in part, by a National Institutes of Health grant (CA133086 and RR023976) to CL.

\section{DISCLOSURE/CONFLICT OF INTEREST}

The authors declare no conflict of interest.

1. Jemal A, Murray T, Ward E, et al. Cancer Statistics CA Cancer J Clin 2005;55:10-30.

2. Frilling $A$, Malago $M$, Testa $G$, et al. Liver transplantation for metastasized extragastrointestinal stromal tumor: a case report and an overview of literature. Transplant Proc 2010;42:3843-3848.

3. Panka DJ, Wang W, Atkins MB, et al. The Raf inhibitor BAY 43-9006 (Sorafenib) induces caspase-independent apoptosis in melanoma cells. Cancer Res 2006;66:1611-1619.

4. Winkler J, Rech D, Kallert S, et al. Sorafenib induces sustained molecular remission in FLT3-ITD positive AML with relapse after second allogeneic stem cell transplantation without exacerbation of acute GVHD: a case report. Leuk Res 2010;34:e270-e272.

5. Guida T, Anaganti S, Provitera L, et al. Sorafenib inhibits imatinibresistant KIT and platelet-derived growth factor receptor beta gatekeeper mutants. Clin Cancer Res 2007;13:3363-3369.

6. Keswani RN, Chumsangsri A, Mustafi R, et al. Sorafenib inhibits MAPKmediated proliferation in a Barrett's esophageal adenocarcinoma cell line. Dis Esophagus 2008;21:514-521.

7. Busse A, Asemissen AM, Nonnenmacher A, et al. Immunomodulatory effects of sorafenib on peripheral immune effector cells in metastatic renal cell carcinoma. Eur J Cancer 2011;47:690-696.

8. Adnane L, Trail PA, Taylor I, et al. Sorafenib (BAY 43-9006, Nexavar), a dual-action inhibitor that targets RAF/MEK/ERK pathway in tumor cells and tyrosine kinases VEGFR/PDGFR in tumor vasculature. Methods Enzymol 2006;407:597-612.

9. Wilhelm SM, Adnane L, Newell $P$, et al. Preclinical overview of sorafenib, a multikinase inhibitor that targets both Raf and VEGF and PDGF receptor tyrosine kinase signaling. Mol Cancer Ther 2008;7: 3129-3140.

10. Ou DL, Shen YC, Yu SL, et al. Induction of DNA damage-inducible gene GADD45beta contributes to sorafenib-induced apoptosis in hepatocellular carcinoma cells. Cancer Res 2010;70:9309-9318.

11. Ou DL, Shen YC, Yu SL, et al. The Bcl-xL inhibitor, ABT-737, efficiently induces apoptosis and suppresses growth of hepatoma cells in combination with sorafenib. Hepatology 2010;52:1310-1321.

12. Shimizu $\mathrm{S}$, Takehara $\mathrm{T}$, Hikita $\mathrm{H}$, et al. The let-7 family of microRNAs inhibits $\mathrm{BCl}-\mathrm{xL}$ expression and potentiates sorafenib-induced apoptosis in human hepatocellular carcinoma. J Hepatol 2010;52: 698-704.

13. Villanueva A, Llovet JM. Targeted therapies for hepatocellular carcinoma. Gastroenterology 2011;140:1410-1426.

14. Roberts LR. Sorafenib in liver cancer-just the beginning. N Engl J Med 2008;359:420-422.

15. Kim R, Byrne MT, Tan A, et al. What is the indication for sorafenib in hepatocellular carcinoma? A clinical challenge. Oncology 2011;25:295.

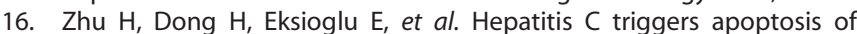
newly developed hepatoma cell line through anti-viral defense system. Gastroenterology 2007;133:1649-1659.

17. Ozawa K, Kitamura O, Yamaoka Y, et al. Hepatic cellular responses to liver cancer: abnormalities in metabolism of mitochondria isolated from human liver involved with carcinoma. Ann Surg 1974; 179:79-87.

18. Inaba K. Nucleic acids and protein synthesis in cancer cell mitochondria. II. Amino acid incorporation into proteins of rat liver and hepatoma cell mitochondria. Acta Med Okayama 1968;22:101-112.

19. Huang P, Yu T, Yoon Y. Mitochondrial clustering induced by overexpression of the mitochondrial fusion protein Mfn2 causes mitochondrial dysfunction and cell death. Eur J Cell Biol 2007;86: 289-302.

20. Zhang E, Zhang C, Su Y, et al. Newly developed strategies for multifunctional mitochondria-targeted agents in cancer therapy. Drug Discov Today 2011;16:140-146.

21. Cheung EC, Joza N, Steenaart NA, et al. Dissociating the dual roles of apoptosis-inducing factor in maintaining mitochondrial structure and apoptosis. EMBO J 2006;25:4061-4073.

22. Chan DC. Mitochondria: dynamic organelles in disease, aging, and development. Cell 2006;125:1241-1252.

23. Westermann B. Mitochondrial dynamics in model organisms: what yeasts, worms and flies have taught us about fusion and fission of mitochondria. Semin Cell Dev Biol 2010;21:542-549.

24. Wenner CE. Targeting mitochondria as a therapeutic target in cancer. J Cell Physiol 2012;227:450-456.

25. Yang F, Brown C, Buettner R, et al. Sorafenib induces growth arrest and apoptosis of human glioblastoma cells through the dephosphorylation of signal transducers and activators of transcription 3. Mol Cancer Ther 2010;9:953-962.

26. Lei X, Chen Y, Du G. Gossypol induces Bax/Bak-independent activation of apoptosis and cytochrome $c$ release via a conformational change in Bcl-2. FASEB J 2006;20:2147-2149.

27. Tian C, Erdmann N, Zhao J, et al. HIV-infected macrophages mediate neuronal apoptosis through mitochondrial glutaminase. J Neurochem 2008;105:994-1005.

28. Tian C, Murrin LC, Zheng JC. Mitochondrial fragmentation is involved in methamphetamine-induced cell death in rat hippocampal neural progenitor cells. PLoS One 2009;4:e5546.

29. Herrmann M, Lorenz HM, Voll $\mathrm{R}$, et al. A rapid and simple method for the isolation of apoptotic DNA fragments. Nucleic Acids Res 1994; 22:5506-5507.

30. Zheng $\mathrm{Y}$, Yamaguchi $\mathrm{H}$, Tian $\mathrm{C}$, et al. Arsenic trioxide $(\mathrm{As}(2) \mathrm{O}(3))$ induces apoptosis through activation of Bax in hematopoietic cells. Oncogene 2005;24:3339-3347.

31. Ramakrishnan V, Timm M, Haug JL, et al. Sorafenib, a dual Raf kinase/ vascular endothelial growth factor receptor inhibitor has significant anti-myeloma activity and synergizes with common anti-myeloma drugs. Oncogene 2010;29:1190-1202.

32. Yu C, Friday BB, Lai JP, et al. Cytotoxic synergy between the multikinase inhibitor sorafenib and the proteasome inhibitor bortezomib in vitro: induction of apoptosis through Akt and c-Jun NH2-terminal kinase pathways. Mol Cancer Ther 2006;5:2378-2387.

33. Polyakov VY, Soukhomlinova MY, Fais D. Fusion, fragmentation, and fission of mitochondria. Biochemistry (Mosc) 2003;68:838-849.

34. Borentain P, Gregoire $\mathrm{E}$, Hardwigsen J, et al. Liver transplantation in a patient treated by sorafenib for hepatocellular carcinoma. Clin Res Hepatol Gastroenterol 2011;35:234-236.

35. Sacco R, Bargellini I, Gianluigi G, et al. Complete response for advanced liver cancer during sorafenib therapy: case report. BMC Gastroenterol 2011;11:4.

36. Roderburg C, Bubenzer J, Spannbauer $M$, et al. Long-term survival of a HCC-patient with severe liver dysfunction treated with sorafenib. World J Hepatol 2010;2:239-242. 
37. Takezawa K, Okamoto I, Yonesaka K, et al. Sorafenib inhibits non-small cell lung cancer cell growth by targeting B-RAF in KRAS wild-type cells and C-RAF in KRAS mutant cells. Cancer Res 2009;69:6515-6521.

38. Yokoyama $\mathrm{H}$, Lundqvist $\mathrm{A}$, Su $\mathrm{S}$, et al. Toxic effects of sorafenib when given early after allogeneic hematopoietic stem cell transplantation. Blood 2010;116:2858-2859.

39. Gedaly R, Angulo P, Hundley J, et al. PI-103 and sorafenib inhibit hepatocellular carcinoma cell proliferation by blocking Ras/Raf/MAPK and PI3K/AKT/mTOR pathways. Anticancer Res 2010;30:4951-4958.

40. Chai $\mathrm{H}$, Luo $A Z$, Weerasinghe $P$, et al. Sorafenib downregulates ERK/ Akt and STAT3 survival pathways and induces apoptosis in a human neuroblastoma cell line. Int J Clin Exp Pathol 2010;3:408-415.

41. Chen KF, Yu HC, Liu TH, et al. Synergistic interactions between sorafenib and bortezomib in hepatocellular carcinoma involve PP2Adependent Akt inactivation. J Hepatol 2010;52:88-95.

42. Rosato RR, Almenara JA, Coe $\mathrm{S}$, et al. The multikinase inhibitor sorafenib potentiates TRAIL lethality in human leukemia cells in association with Mcl-1 and CFLIPL down-regulation. Cancer Res 2007;67:9490-9500.

43. Lierman $\mathrm{E}$, Lahortiga I, Van $\mathrm{MH}$, et al. The ability of sorafenib to inhibit oncogenic PDGFRbeta and FLT3 mutants and overcome resistance to other small molecule inhibitors. Haematologica 2007;92:27-34.

44. Semenzato M, Cogliati S, Scorrano L. Prohibitin(g) cancer: aurilide and killing by OPA1-dependent cristae remodelling. Chem Biol 2011;18:8-9.

45. Kahatri S, Plas DR. Targeting bioenergetics to enhance cancer chemotherapy. Cancer Biol Ther 2009;8:1659-1661.

46. Merkwirth C, Dargazanli S, Tatsuta T, et al. Prohibitins control cell proliferation and apoptosis by regulating OPA1-dependent cristae morphogenesis in mitochondria. Genes Dev 2008;22:476-488.

47. Piao L, Li Y, Kim SJ, et al. Regulation of OPA1-mediated mitochondrial fusion by leucine zipper/EF-hand-containing transmembrane protein1 plays a role in apoptosis. Cell Signal 2009;21:767-777.

48. Chen L, Gong Q, Stice JP, et al. Mitochondrial OPA1, apoptosis, and heart failure. Cardiovasc Res 2009;84:91-99. 